\title{
Ganancia auditiva en timpanoplastía tipo I, Hospital de Especialidades №2 del Centro Médico Nacional del Noroeste "Lic. Luis Donaldo Colosio Murrieta"
}

\author{
Enrique Gómez Rodriguez ${ }^{1}$, Lugo Machado Juan Antonio ${ }^{2}$, Calderón Celina ${ }^{3}$
}

\section{RESUMEN}

Objetivo: Determinar si existe ganancia auditiva posterior a la realización de timpanoplastía tipo 1 en pacientes con otitis media crónica e hipoacusia conductiva.

Material y Métodos: Este estudio se realizó en servicio de otorrinolaringología del Hospital de especialidades $\mathrm{N}^{\circ} 2$, unidad médica de alta especialidad, centro médico nacional del noroeste en ciudad Obregón, Sonora. Se llevó a cabo un estudio de cohorte retrospectivo, analítico, longitudinal. Descripción general del estudio. Se revisó el expediente clínicos de los pacientes con diagnóstico de secuelas de otitis media crónica, posoperados de timpanoplastía tipo 1, atendidos en el servicio de esta institución de marzo 2011 a abril 2014. Se tomaron datos como edad, sexo, lugar de procedencia, si existieron complicaciones postquirúrgicas, nivel promedio de audición previo y posterior a intervención quirúrgica.

Resultados: De un total de 237 expedientes revisados, se encontraron 51 expedientes completos, de los cuales 33 pacientes fueron femeninos (64.7\%) el rango de edad fue de 9 a 71 años, con media de 36.7 años. el lado operado más frecuente fue el derecho en 33 pacientes (64.7\%). No se documentó ninguna complicación en los expedientes revisados. La evaluación de la audiometría previa la cirugía, se observó algún grado de hipoacusia en 46 pacientes (90.19\%) y normal en 5 pacientes (9.8\%). Postquirúrgicamente se mostró audiometría normal en 38 pacientes (74.5\%), y anormal en 13 pacientes $(25.49 \%)$ con una $\mathrm{p}<.0001$. Existe ganancia auditiva estadísticamente significativa con timpanoplastía tipo 1 en pacientes con secuelas de otitis media crónica en la unidad médica alta especialidad, cd. Obregón, Sonora.

Conclusión: En la mayoría de nuestros pacientes la timpanoplastía tipo 1, presentaron mejoría en la audición. Estos resultados son similares a los que encontraron otros autores como shrestha y et al.

Palabras clave: otitis media crónica, timpanoplastía, ganancia de audición.

\section{Hearing gain in tympanoplasty type I , Lic. Luis Donaldo Colosio Murrieta Northwest Specialty Hospital №2}

Objective: To determine if there is a hearing gain after performing a tympanoplasty type 1 in patients with mid level chronic otitis and conductive hearing loss.

Material and Methods: This study was conducted in the otolaryngology service of the Specialty Hospital $\mathrm{N}^{\circ} 2$, highly specialized medical unit, Northwest National Medical Center in Obregon city, Sonora. A study of a retrospective, analytical and longuitudinal cohort was carried out. Overview of the Study: Clinical records of patients diagnosed with sequale of mid level chronic otitis, tympanoplasty type 1 post surgery patients in this institution from March 2011 to April 2014 were reviewed. Data such age, sex and place of origin were taken and if there were any post surgery complications, average level of hearing previous and after surgery.

Results: From a total of 237 reviewed patients, 51 were completed, of which 33 were female (64.7\%), range of age was fron 9 to 71 years old with an average of 36.7 years old. The most frequent operated side was the right in 33 patients (64.7\%). No complications in the files reviewed were found. In the evaluation of audiometry previous the surgery, certain degree of hearing loss was observed in 46 patients $(90.19 \%)$ and normal in 5 patients $(9.8 \%)$. Postsurgically an standard audiometry were foun in 38 patients $(74.5 \%)$ and anormal in 13 patients $(25.49 \%)$ with $p<.0001$. There is a statistically significant hearing gain with tympanoplasty type 1 in patients with sequale of mid level chronic level in the highly specialized medical unit, Northwest National Medical Center in Obregon city, Sonora.

Conclusions: In most of our patients tympanoplasty type 1 , there was an improvement in hearing. This results are similar to those found in authors such as Shrestha and et al.

Key words: mid level chronic otitis, tympanoplasty, hearing gain.

1. Médico Residente de Otorrinolaringología del Hospital de Especialidades No.2 “Luis Donaldo Colosio Murrieta”, Unidad Médica de Alta Especialidad, CMNNO, Cd Obregón, Sonora.

2. Médico adscritoal Servicio de Otorrinolaringología del Hospital de Especialidades No.2 “Luis Donaldo Colosio Murrieta”, Unidad Médica de Alta Especialidad, CMNNO, Cd Obregón, Sonora.

3. Médico adscrito al Servicio de Audiología y Otoneurología del Hospital de Especialidades No.2 “Luis Donaldo Colosio Murrieta”, Unidad Médica de Alta Especialidad, CMNNO, Cd Obregón, Sonora. 


\section{INTRODUCCIÓN}

La otitis media crónica supurativa es una enfermedad mundial caracterizada por hipoacusia y otorrea que causa un impacto financiero en la sociedad y psicológico importante para el paciente (1). La principal secuela que deja la otitis media crónica es la perforación timpánica residual, la cual ocasiona una comunicación entre el oído medio y externo, siendo más propenso a reinfectarse y complicarse; secundariamente la audición se disminuye en estos casos (1).

El sonido es trasmitido hacia el oído medio por conducción aérea y ósea. La conducción aérea consiste en el oído externo, membrana timpánica y huesecillos del oído medio. De hecho se involucran otros mecanismos con rol notable en la audición: la conducción aérea y otros 5 factores que se consideran como parámetros efectivos en la conducción, que incluyen: canal auditivo externo, inercia de los huesecillos del oído medio, inercia de los fluidos cocleares, cambios en espacio coclear y fluido cerebroespinal trasmitido por presión (2). El rol del canal auditivo externo es más obvio en las frecuencias por debajo de $1000 \mathrm{hz}$. La inercia de los huesecillo del oído medio probablemente es más efectiva en alta frecuencia (2).

El conducto auditivo externo ausente o patológico ha demostrado ser responsable de una pérdida de audición de hasta $15 \mathrm{db}$ únicamente, sin embargo la falta de integridad de membrana timpánica puede causar hipoacusia de hasta 35-40 db adicionales, en caso de que la hipoacusia sea mayor de $40 \mathrm{db}$, no sólo la membrana timpánica estaría involucrada, sino también cualquier factor dentro de la conducción auditiva, incluyendo huesecillos y ventana oval, por ejemplo; y si es mayor a $60 \mathrm{db}$ ya habría un daño neurosensorial(3).

La audiometría de tonos puros es un estudio en donde se mide objetivamente la respuesta del paciente a sonidos pasados a diferentes frecuencias y a diferentes decibeles, graficando así diferentes resultados y midiendo el nivel de audición. Por frecuencia y lo valorado en otros estudios como en el de sharakuman et al, se puede realizar previo a la cirugía y 3 meses después de la cirugía, donde la ganancia de $9 \mathrm{db}$ en la audición se reporta como ganancia (4).

La timpanoplastía es una técnica para sellar membrana timpánica, teniendo como principal objetivo evitar complicaciones y como segundo objetivo mejorar la audición y dejar libre de enfermedad el oído medio (5). La otitis media crónica es la principal indicación para timpanoplastía (6). Esta técnica se introdujo por Wullstein y Zollner, los cuales publicaron una clasificación enfocada en el tipo de reconstrucción de cadena osicular que se necesitara. Los cinco tipos de timpanoplastía se refieren a la estructura más lateral que se encuentra intacta en la cual el mecanismo de la conducción se pueda reconstruir (7). La tipo I indica cuando los 3 huesecillos están presentes y móviles. Además la reconstrucción ósea no es necesaria (8). La tipo II se coloca injerto hacia un yunque y estribo intactos (9). La tipo III existe cuando la supra estructura del estapedio esta móvil e intacta (10). La tipo 4 se describe como ausencia de supra estructura, por lo que el injerto se recuesta sobre la platina del estribo. La tipo 5 se refiere a la fenestra creada en canal semicircular horizontal (11). La timpanoplastía tipo 1 se realiza en caso de perforaciones centrales secas (12). La timpanoplastía con mastoidectomía está indicada cuando la mucosa de oído esta congestionada, polipoidea, húmeda o con descarga (13).

La timpanoplastía es el procedimiento de elección para el tratamiento de secuelas de otitis media crónica simple (14). El principal objetivo es erradicar la enfermedad de oído medio y restaurar mecanismos de conducción, incluyendo membrana timpánica y huesecillos. La principal indicación es en perforaciones timpánicas sin reparación espontánea y que presentan pérdida auditiva, sin embargo el tinnitus no está claro que mejore posterior a procedimiento (15).

Unos de los objetivos secundarios en la cirugía de otitis media crónica es la restauración de la audición (15). Como sea, los resultados han variado de un estudio a otro, y la cirugía del oído crónicamente infectado muestra diferentes resultados y pronóstico de acuerdo a varios factores (ejemplo: presencia y extensión de colesteatoma, severidad de enfermedad de la mucosa, métodos de manejo para la mastoides y métodos para la reconstrucción acicular) $(14,15)$. Los estudios de audición con frecuencias específicas son importantes porque el nivel de audición en tonos puros no siempre resulta con el mismo patrón que la percepción de la audición $(14,15)$. Solamente existe un estudio previo comparando audición preoperatoria y postoperatoria posterior a timpanoplastía hecho por Sharankumar Shetty et al., donde se estudiaron a 50 pacientes con 
realización de timpanoplastía, donde en promedio hubo ganancia de 15.2 a $30 \mathrm{db}$, de los cuales $76 \%$ fueron por timpanoplastia tipo 1, 16\% por tipo 2 y $10 \%$ por tipo $3.86 \%$ del total de los pacientes restauraron audición normal.

Existe otro estudio hecho por shrestha et al (9), donde comparan audición postquirúrgica pero en una miringoplastía, solo reportaron 2 casos, siendo no confiable ni concluyente.

Nuestro estudio se centra en evaluar la mejoría de audición, con audiometría pre y postoperatoria, en las secuelas de otitis media crónica simple posterior a timpanoplastía tipo 1.

Las perforaciones timpánicas son secuelas de otitis media crónica, las cuales producen hipoacusia y pueden afectar calidad de vida del paciente. la timpanoplastía tipo 1 es el procedimiento de elección para perforaciones timpánicas centrales sin afección a cadena osicular que tiene como objetivo principal; la corrección anatómica del defecto para evitar las consecuencias por la falta del mismo, que son las infecciones recurrentes, sin embargo no ha sido evaluado un efecto secundario que es la recuperación auditiva del factor conductiva por lo que es importante conocer el nivel de audición pre y postquirúrgico, con una audiometría tonal para evaluar el impacto. Existen beneficios potenciales posteriores a la cirugía correctiva de la membrana timpánica, ya que no solo el paciente mejora la audición y calidad de vida subsecuentemente, sino también el instituto se beneficia al contar con evidencia de la funcionalidad de la timpanoplastía. Solamente se ha reportado un estudio internacional hecho por Sharankumar Shetty et al., sin haber estudios previos en la institución o la nación, lo cual lo convierte en un estudio original nacionalmente, con adecuada factibilidad ética y económica.

Las secuelas de otitis media crónica, como la perforación timpánica, tiene impacto importante en la audición, con repercusiones y consecuencias graves en la calidad de vida y desempeño laboral de cada individuo, así como a nivel institucional generando gastos económicos en consultas, estudios y tratamientos médicos continuos y repetitivos, por lo que es necesario la realización del estudio al valorar el impacto posterior a procedimiento quirúrgico, así como en la prevención de complicaciones para que no progrese la hipoacusia.

Nuestro objetivo fue determinar si existe ganancia auditiva, comprobada por audiometría, en pacientes pos operada de timpanoplastía tipo 1 con secuelas de otitis media crónica.

\section{MATERIAL Y MÉTODOS}

Se llevó a cabo un estudio de cohorte retrospectivo, analítico y longitudinal. Se incluyeron los expedientes de los pacientes pos operados de timpanoplastía tipo 1 por secuelas de otitis media crónica atendidos en el servicio de otorrinolaringología, unidad médica de alta especialidad, hospital de especialidades $\mathrm{N}^{\circ} 2$ del centro médico nacional del noroeste "Lic. Luis Donaldo Colosio Murrieta", Cd. Obregón. Sonora el estudio se realizó durante el mes de marzo 2011 a abril 2014, ya que los expedientes previos a marzo 2011 se depuraron y no hay archivos previos de estudios o de cirugías realizadas.

Se incluyeron expedientes completos con audiometría pre y postquirúrgica de pacientes con diagnóstico de secuelas de otitis media crónica por perforación timpánica de tipo central, pos operado de timpanoplastía tipo 1 , la cual se realizó bajo anestesia general, con abordaje retro auricular y toma de fascia del músculo temporal ipsilateral. Se excluyeron pacientes que se les realizó otro procedimiento en oído como mastoidectomía, osiculoplastía y/o estapedectomía, pacientes con rechazo de injerto o con perforación timpánica residual durante cualquier momento de su evolución, pacientes con otros tipos de timpanoplastía que no sea la tipo 1 , pacientes con trauma acústico o perforación timpánica traumática o pacientes con hipoacusia sensorial o severa, profunda, restos o anacusia y expedientes incompletos. Se evaluaron las audiometrías y se obtuvo un promedio de decibeles (db) en todas las frecuencias auditivas para cada pacientes, tanto previo a la intervención quirúrgica como posterior a ésta.

La información se capturó en una base de datos utilizando el paquete estadístico SPSS para Mac versión 21 , en el cual se realizó el análisis estadístico y se interpretaron los resultados. Se utilizó estadístico descriptivo con frecuencias y porcentajes para las variables cualitativas, así como medidas de tendencia central como media o mediana para variables cuantitativas. Para medidas de dispersión se utilizó desviación estándar, mínima, máxima y rango para distribución normal. Se utilizó prueba no paramétrica de test de wilcoxon y chi cuadrada, con rango de confianza de $95 \%$ y se consideró la $p<.0001$ como estadísticamente significativa. 


\section{RESULTADOS}

De un total de 237 expedientes revisados, se encontraron 51 expedientes completos. De los cuales 33 pacientes fueron femeninos (64.7\%) y 18 (35.3\%) fueron masculinos (Tabla 1), el lado operado más frecuente fue el derecho en 33 pacientes $(64.7 \%)$ y 18 de lado izquierdo (35.3\%) (Tabla 2), no se documentó ninguna complicación en los expedientes revisados, el rango de edad fue de 9 a 71 años, con media de 36.7 años.

En cuanto al lugar de procedencia de los pacientes por entidad federativa, hubo mayor aporte del estado de Sonora con 34 pacientes (66.6\%) comparado con el estado de Sinaloa con 17 pacientes (33.3\%) (Tabla 3).

De los 51 expedientes revisados, en la audiometría pre quirúrgica se observó nivel de audición normal (20db) en 5 pacientes (9.8\%), audición en $30 \mathrm{db}$ en 10 pacientes $(19.6 \%)$, en $40 \mathrm{db}$ se observó en 28 pacientes (54.7\%) y en $50 \mathrm{db}$ en 8 pacientes (15.7\%), en cuanto la audiometría posterior a la cirugía se observó 1 paciente con audición en $10 \mathrm{db}(2 \%), 37$ pacientes con audición de $20 \mathrm{db}(72.5 \%), 11$ pacientes con audición en $30 \mathrm{db}(21.6 \%)$, y 2 pacientes con audición de $40 \mathrm{db}$ (3.9\%) (Figura 1).

Definiendo una audiometría normal, como un promedio de audición de $20 \mathrm{db}$ o menos, y anormal con un promedio de audición de $30 \mathrm{db}$ o más, se mostró la distribución de la siguiente forma: prequirugicamente se observó audiometría anormal en 46 pacientes $(90.19 \%)$ y normal en 5 pacientes (9.8\%) (Figura 2). Postquirúrgicamente se mostró audiometría normal en 38 pacientes $(74.5 \%)$, y anormal en 13 pacientes $(25.49 \%)$ con una $\mathrm{p}<.0001$ (Figura 3).

Tabla 1. Frecuencia y porcentaje de distribución de género

\begin{tabular}{|c|c|c|}
\hline Sexo & $\mathrm{n}=$ & Porcentaje \\
\hline Femenino & 33 & $64.7 \%$ \\
\hline Masculino & 18 & $35.3 \%$ \\
\hline Total & 51 & $100 \%$ \\
\hline
\end{tabular}

Tabla 2. Frecuencia y porcentaje de lado operado de Timpanoplastía tipo 1

\begin{tabular}{|l|c|c|}
\hline Lado operado & $\mathrm{n}=$ & Porcentaje \\
\hline Derecho & 33 & $64.7 \%$ \\
\hline Izquierdo & 18 & $35.3 \%$ \\
\hline Total & 51 & $100 \%$ \\
\hline
\end{tabular}

Tabla 3. Tabla procedencia de pacientes pos operados de timpanoplastía tipo 1

\begin{tabular}{|c|c|c|}
\hline Entidad federativa & $\mathrm{n}=$ & Porcentaje \\
\hline Sonora & 34 & $66.6 \%$ \\
\hline Sinaloa & 17 & $33.3 \%$ \\
\hline Total & 51 & $100 \%$ \\
\hline
\end{tabular}


Ganancia auditiva en timpanoplastía tipo I, Hospital de Especialidades №2

del Centro Médico Nacional del Noroeste "Lic. Luis Donaldo Colosio Murrieta"

Figura 1. Porcentajes de pacientes antes (pre quirúrgico) y después (postquirúrgico) de la timpanoplastía tipo 1 divididos por decibeles (db) promedio en audiometría

Ganancia auditiva en timpanoplastía tipo I, Hospital de Especialidades №2 del Centro Médico Nacional del Noroeste "Lic. Luis Donaldo Colosio Murrieta"

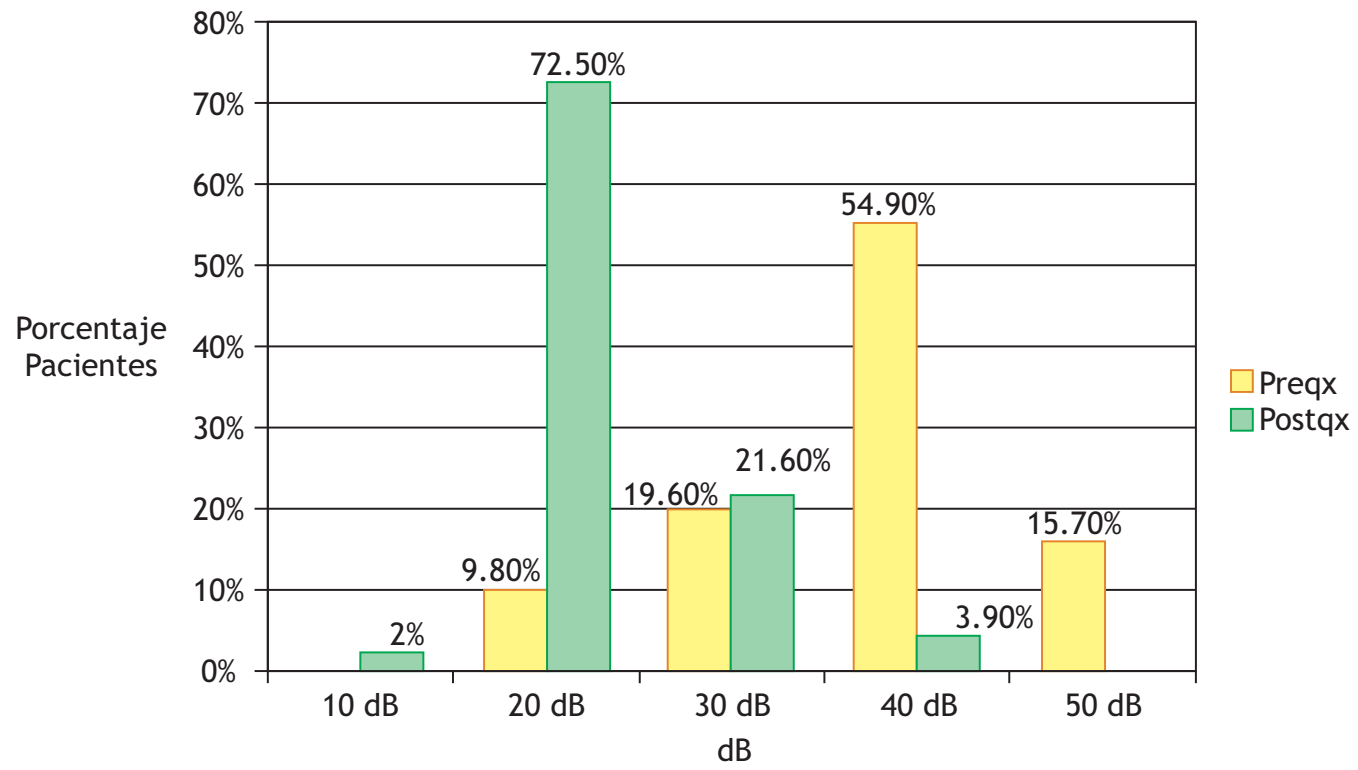

Figura 2. Porcentaje de pacientes con hipoacusia y normoacusia previo a timpanoplastía tipo 1

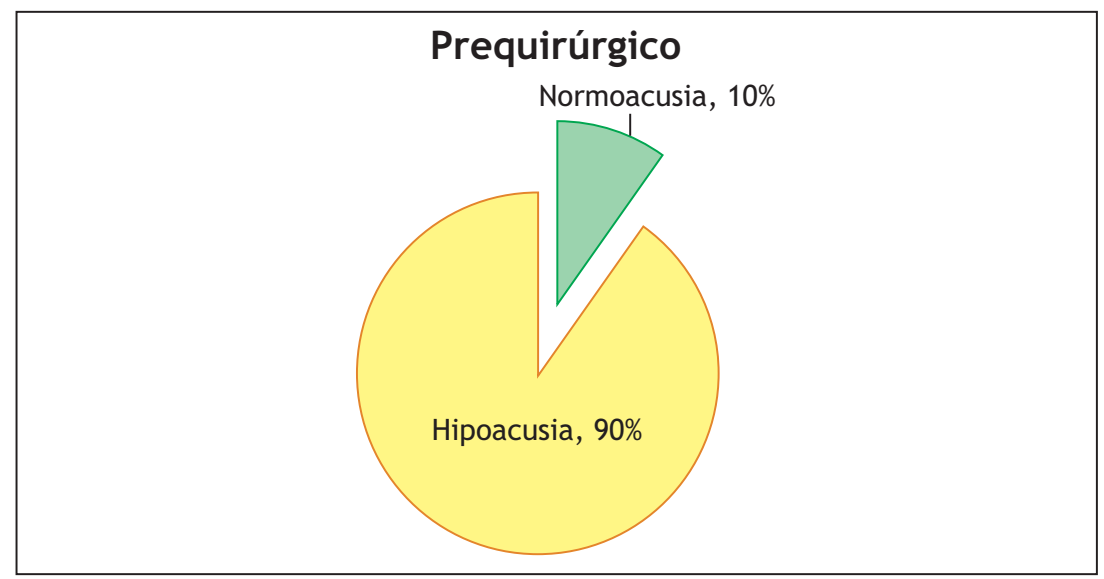

Figura 3. Porcentaje de pacientes con normoacusia e hipoacusia posterior a timpanoplastía tipo 1

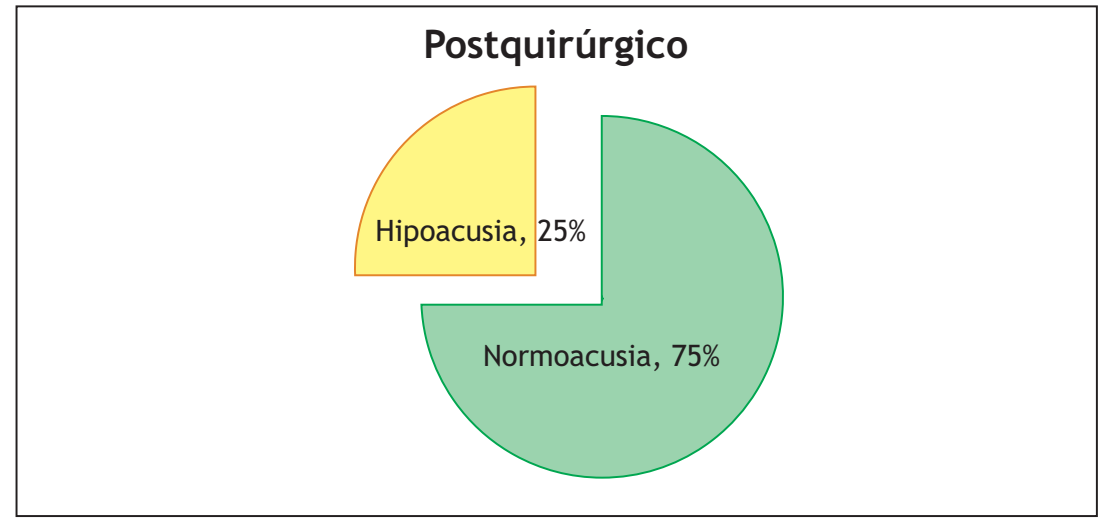




\section{DISCUSIÓN}

Los hallazgos encontrados en este estudio muestran que la realización de la timpanoplastía es segura y aunque el principal objetivo de la timpanoplastía no es la mejoría de audición, si no mantener el oído seco y seguro, con éste estudio se comprueba que se puede mejorar la audición al momento de realizar este tipo de cirugía para pacientes con secuelas de otitis media crónica. Este estudio se limitó a estudiar la ganancia auditiva en este tipo de pacientes con injerto ya integrado.

En nuestro estudio se incluyeron 51 pacientes, lo cual muestra resultados similares lo encontrado por Sharankumar (1) y cols; sin embargo en la distribución por sexo nosotros si encontramos diferencia significativa, en la que Sharankumar no presentó diferencia entre sexo, en nuestro estudio hubo predominancia del sexo femenino contra el masculino $(64.7 \%$ contra $35.3 \%)$ una similitud con el estudio mencionado es la distribución de pacientes en su nivel de audición previa y posterior a la intervención quirúrgica, a pesar de que en el otro estudio miden la audición por el promedio del gap auditivo (diferencia entre vía ósea y vía aérea) y nosotros medimos la audición con un promedio de $\mathrm{db}$ en todas las frecuencias. La distribución de ganancia auditiva fue similar comparando su resultado de $84 \%$ de los pacientes permaneciendo con audición normal frente a un $16 \%$ con hipoacusia, a diferencia con nuestro resultado que mostró $75 \%$ de los pacientes con normoacusia y $25 \%$ con hipoacusia.

Otro estudio obtuvo ganancia auditiva en 50 pacientes, con una muestra muy similar a la nuestra, en la cual compararon audición una semana después de una miringoplastía con anestesia local y abordaje endaural, A pesar de que el abordaje y la anestesia fueron diferentes, en nuestra muestra hubo reducción del gap, lo que refleja mejoría de audición al igual que en nuestro estudio (7).

Aunque el estudio hecho por vijayendra (4) y colaboradores es un poco diferente a nuestro estudio ya que analizan audición comparando la realización de canaloplastía, en conjunto con timpanoplastía tipo 1, ellos observaron ganancia auditiva de $9 \mathrm{db}$ únicamente.

En nuestro estudio existen sesgos como el tiempo en que se realizó la audiometría postquirúrgica, ya que al ser un estudio retrospectivo, no existió un consenso del intervalo de tiempo adecuado para realizar una audiometría posterior a la intervención quirúrgica, dejando fuera si existiría algún tipo de modificación en la audición a diferentes tiempos posterior a la cirugía, imposibilitando la oportunidad de realizar audiometrías posteriores. Otra limitación del estudio fue la realización de un promedio de $\mathrm{db}$ en todas las frecuencias, ya que en muchas ocasiones las frecuencias altas pueden necesitar mayores decibeles y las frecuencias bajas necesitan menores decibeles, alterando el promedio en total de cada uno de los pacientes, pero en forma general, se puede valorar el nivel de audición con el promedio de db en todas las frecuencias.

Esta revisión de nuestra serie, es una base para la realización de un estudio prospectivo, valorando audiometría y timpanometría con un intervalo de tiempo estandarizado y a largo plazo, para valorar audición después de años de evolución.

En conclusión, nuestros resultados son muy similares a lo encontrado por otros autores, donde se comprueba que muchos de los pacientes sometidos a timpanoplastía tipo 1, muestran mejoría de la audición, posterior al evento quirúrgico. Podemos concluir que existe ganancia en la audición en pacientes operados de timpanoplastía tipo 1 por secuelas de otitis media crónica y que debe de contemplarse como uno de los objetivos de la cirugía en pacientes seleccionados.

\section{REFERENCIAS BIBLIOGRÁFICAS}

1. Sharankumar Shetty et al. Pre-operative and post-operative assessment of hearing following tympanoplasty. Indian J Otolaryngol Head Neck Surg. 2012 64(4): 377-381.

2. Farhad Mokhtarinejad et al. Ear surgery techniques results on hearing theshold improvement. J Res Med Sci. september 2013; 18(9): 746-749.

3. Hyeog-gi Choi et al. Frequency-specific hearing results after surgery for chronic ear diseases. Clinical and Experimental Otorhinolaryngology. 2011; 4(3): 126-130.

4. H. Vijayendra et al. Comparative study of hearing improvement in type 1 tympanoplasty with and without canalplasty. Head Neck Surg. 2008; 60:341-344.

5. Adriana Da Silva Lima et al. The effect of timpanoplasty on tinnitus in patients with conductive hearing loss: a six month follow-up. Rev Bras Otorrinolaringol. 2007; 73(3) 284-9.

6. Asok K. Saha et al. Evaluation of improvement of hearing in type i tympanoplasty and its influencing factor. Indian Journal of Otolaryngology and Head and Neck Surgery. 2006; 58(3): 33-36

7. Sismanis A et al. Tympanoplasty glasscock-shambaugh 'surgery of the ear', Bc decker inc, Hamilton 2003; 24.

8. Mills RP, Frootko NJ et al. Management of chronic suppurative otitis media, \& reconstruction of the middle ear. Scott brown 6th edition. 1997; vol 3, chapters 10, 11.

9. Shrestha $S$, Sinha BK et al. Hearing results after myringoplasty. Kathmandu Univ Med J. 2006; 4(16): 455-459. 
Ganancia auditiva en timpanoplastía tipo I, Hospital de Especialidades №2

del Centro Médico Nacional del Noroeste "Lic. Luis Donaldo Colosio Murrieta"

10. Bhusal CL, Guragain RPS, Shrivastav RP et al. Correlation of hearing impairment with site of tympanic membrane perforation. BMC Ear Nose Throat Disord. 2009; 4(9):1

11. Yung $M W$ et al. Myringoplasty: hearing gain in relation to perforation site. J Laryngol Otol. 1983; 97:11-17.

12. Ahmed S, Wand Ramani GV et al. Hearing loss in perforations of the tympanic membrane. J Laryngol Otol. 1979; 93:1091-1098

13. Umar AS, Ahmed $Z$ et al. Anatomical and functional outcome following type-1 tympanoplasty in chronic tubotympanic suppurative otitis media. Military Hospital Rawalpindi, combined military hospital multan. 2008; 1:12-13.

14. Yuen Ap, Ho Wk, Hui Y, Wei Wi, Au Dk et al. Correlation of pure tone audiogram results and hearing benefit of tympanoplasty for chronic suppurative otitis media. Ann Otol Rhinol Laryngol. 2000; 109(4):381-384

15. Carhart R. et al. Clinical application of bone conduction audiometry. Arch Otolaryngol. 1950; 51:798-808.
Fuentes de Financiamiento

Este artículo ha sido financiado por los autores.

\section{Conflicto de interés}

Los autores declaran no presentar ningún conflicto de interés.

Correspondencia:

Juan Antonio Lugo Machado

Dirección: Prolongación Hidalgo Bellavista -Cajeme 85130

Ciudad Obregón, Sonora.

Teléfono: +6441559891

Correo electrónico: otorrinox@gmail.com

Recibido: 05 de marzo de 2016

Aprobado: 18 de abril de 2016 